\title{
Logistic regression for simulating damage occurrence on a fruit grading line
}

\author{
C. Bielza , P. Barreiro, M.I. Rodríguez-Galiano , \\ J. Martín
}

Artificial Intelligence Department. School of Computer Science, Madrid Technical University, Campus de Montegancedo stn., 28660 Boadilla del Monte, Madrid. Spain

Rural Engineering Depariment, School of Agricultural Engineering, Madrid Techical University, Madrid, Spain

Mathematics Department, Technical School of Caceres, Unitersity of Extremadura. Caceres, Spain

\begin{abstract}
Many factors influence the incidence of mechanical damage in fruit handled on a grading line. This makes it difficult to address damage estimation from an analytical point of view. During fruit transfer from one element of a grading line to another, damage occurs as a combined effect of machinery roughness and the intrinsic susceptibility of fruit. This paper describes a method to estimate bruise probability by means of logistic regression, using data yiclded by specific laboratory tests. Model accuracy was measured via the statistical significance of its parameters and its classification ability. The prediction model was then linked to a simulation model through which impacts and load levels, similar to those of real grading lines, could be generated. The simulation output sample size was determined to yield reliable estimations. The process makes it possible to derive a suitable line design and the type of fruit that should be handled to maintain bruise levels within European Union (EU) Standards. A real example with peaches was carricd out with the aid of the software implementation simLIN ${ }^{\text {ai }}$, developed by the authors and registered by Madrid Technical University. This kind of tool has been demanded by inter-professional associations and grading lines designers in recent years.
\end{abstract}

Keywords: Bruise probability; Logistic regression; Simulation; Grading lines; Fruit 


\section{Introduction}

A growing demand for higher quality fresh fruit and vegetables has revealed a world-wide problem concerning mechanical damage of products reaching the consumer through the distribution chain. Harvesting, handling, post-harvest treatments, packaging, transport and fruit distribution involve a large number of mechanical operations that subject the produce to dynamic loads, mainly impacts. Mechanical handling on grading lines is one of the most hazardous operations as far as mechanical damage is concerned. International studies demonstrate the high agressiveness of fruit grading lines (Miller and Wagner, 1991; Timm et al., 1991; Sargent et al., 1992; Ortiz-Cañavate et al., 1999). One of the problems to account for the aggressiveness of grading lines is that damages not only appear immediately after grading but up to $48 \mathrm{~h}$ later. These are called latent damages (Prussia et al., 1987), and many times have been referred to as transport damages. Two thirds of the crop could be damaged in grading lines, according to observations in the Murcia region of Spain (National Research Project PTR 94-0082): 20-40\% of damaged fruit with bruises visible just after grading, and $40-60 \%$ of damaged fruit with latent damages.

A scrupulous analysis of grading lines has proved to be useful for locating the elements and intermediate connections, also referred to as transfer points, responsible for such dynamic loads, as well as for finding out how to reduce the damage they cause. There are some reasons why grading lines tend to generate large percentages of damaged fruit (García-Ramos et al., 2002). First, grading lines are customised solutions, which need to fit into a given building. This fact makes them difficult to be compared without specific data. Second, the way transfer points are mounted may be critical. We often find structure elements beneath the falling path, which cause the impact intensity to rise dramatically. Third, the maintenance of grading lines is deficient most of the times. This fact leads to situations where the padding materials are deteriorated or have been substituted without enough knowledge on the subject, including more elastic material than energy absorbing ones.

The high fruit handling rate of a grading line-typically $15 \mathrm{t} / \mathrm{h}$ for a $1.5 \mathrm{~m}$-wide line-rules out on-line testing of gradual improvements unless they have previously proved to be successful. Despite efforts to establish algorithms to evaluate grading lines in relation to mechanical damage (Barreiro et al., 1997), the complexity of grouping the aspects of a fruit-grading line system in a mathematical model has prevented the achievement of analytical solutions and/or the development of realistic simulations. Wide interprofessional associations such as CTIFL (http:/www,ctifl.fr) or VCBT (http:/www.agr.kuleuven.ac.be/aee/vebt) and grading line designers, are major applicants for decision support systems on damage reduction in fruit grading lines. The Physical Properties Laboratory (LPF) of the School of Agricultural Engineering at Madrid Technical University makes consultant work on the subject.

In this paper, we use logistic regression to estimate bruise probability on a fruit grading line. Some researchers also used logistic regression for bruise probability estimation but in a very different way. Bollen and Cox (1991) predicted the probability of bruising with regard to a particular transfer point. They first 
determined bruise probabilities as a function of the impact characterised by drop height ( $\mathrm{mm}$ ) at the transfer point, fitting a logistic function. To do so, they used data obtained in the laboratory when dropping fruit from a range of selected heights onto selected surfaces (typical impact surfaces used on grading lines). Bollen (1993) used the same approach, considering the impact characterised by its energy instead of the drop height at the transfer point.

To estimate the final bruise probability for a fruit crossing a certain transfer point, these authors (Bollen and Cox, 1991; Bollen, 1993) assumed that the number of bruises at the transfer point are distributed according to a Poisson distribution. Then, the probability that a fruit is bruised for an isolated transfer point is the probability that the number of bruises do not equal zero. However, we need the bruise probability not only for a particular transfer point but also for simulating the whole grading line (elements and transfer points travelled). Moreover, besides impact, it is essential that the model considers the inherent fruit susceptibility to bruise occurrence.

The research presented in this paper includes four primary objectives. These were: first, to predict fruit bruise probabilities given a data set of measurable fruit characteristics; second, to characterise bruise probabilities as an appropriate set of distribution parameters for a particular fruit species or variety; third, to characterise industrial fruit grading lines as a database of rules and constraints and fourth, to simulate the behaviour and interaction between fruit and industrial grading lines. The methodology is illustrated and discussed through a real example.

\section{Background}

A grading line machine is a series of elements, placed in a well-defined order, which manipulate and prepare harvested or stored fruits for marketing. It is typically composed of specific elements (one per line) such as dumper, sizer, packaging area, which are connected by generic elements, including conveyors, rollers and chutes. Each element can take a different form, e.g. twist chain dumper versus box dumper, with very different levels of aggression. The transfer points between two consecutive elements, either specific-generic or generic-generic are the main cause of impacts in a grading line. The number and type of transfer points vary from line to line, since they are designed to fit the configuration of the building in which the grading line will be installed. Thus, there are rarely two identical grading lines, leading to the need for evaluating the performance of individual cases.

Fruit bruising, the main type of mechanical damage, occurs as an overall effect of two combined factors: machine roughness and intrinsic fruit susceptibility (Barreiro et al., 1997).

Electronic fruits, similar to the fruit in size and shape, are used in grading lines to assess machine roughness (García et al., 1996). They can locate critical points in grading lines, characterising them by several parameters. All impacts are labelled by their intensity in terms of deceleration units $\left(g \times \mathrm{m} / \mathrm{s}^{2}\right.$, where $g$ refers to gravity acceleration) and the type of surface responsible for the impact of the fruit 
(characterised by velocity change in $\mathrm{m} / \mathrm{s}$ ). When an electronic fruit is handled repeatedly on a grading line, the impact distribution at each transfer point, TP, may be estimated. The accuracy of the experimental data for bruise probability depends on the number of runs of the electronic fruit along the line, 14 for the data cited by Garcia et al. (2000). So far, databases concerning the roughness of specific transfer points in commercial grading lines are available. These databases use impact intensity discretised in ranges for the characterisation of each transfer point. For example, if we consider four different intervals, we could get: $P$ (TP yields impact $\leq$ $50 \mathrm{~g})=0.6, P(\mathrm{TP}$ yields $50 \mathrm{~g}<$ impact $\leq 100 \mathrm{~g})=0.2, P(\mathrm{TP}$ yields $100 \mathrm{~g}<$ impact $\leq$ $150 \mathrm{~g})=0.1, P($ TP yields $150 \mathrm{~g}<$ impact $\leq 200 \mathrm{~g})=0.1$. Values above $200 \mathrm{~g}$ should never be achieved in a grading line. These intervals have been proposed in Miller and Wagner (1991).

Intrinsic fruit bruise susceptibility can be assessed by means of laboratory tests (quasi-static and dynamic loading). Well-known relationships between bruise susceptibility and physical and rheological properties of fruit exist and are outlined by García and Ruiz-Altisent (1997). Thus, bruise susceptibility increases for lower curvature radius of surfaces in contact, higher tissue turgidity, higher visco-elastic behaviour, lower fruit firmness, etc. Yet, by no means all the sources of variation related to bruise susceptibility have been identified. Furthermore, bruise susceptibility is heavily dependent on particular varieties and harvest and post-harvest treatments.

Modelling bruise susceptibility consists of obtaining empirical relationships between a bruise characterisation measure (dependent variable) and different quantities (independent variables) taken from the above laboratory tests on a variety-based approach. A proper selection of the bruise characterisation measure is basic, and it depends heavily upon the behaviour of fruit. In many cases bruise volume $\left(\mathrm{mm}^{3}\right)$ has been used for modelling bruise susceptibility (Kampp and Pedersen, 1990; Chen and Yazdani, 1991; Jones and Holt, 1991). However, the unpredictable behaviour of many species and varieties for the onset of bruising, rather points to bruise probability as the best characteristic for modelling bruise susceptibility in these cases (García et al., 2000).

Most of the instrumental variables used for modelling bruise susceptibility have been summarised (see García et al., 2000) as: Load Level $\left(\mathrm{N}\right.$ or $\mathrm{m} / \mathrm{s}^{2}$, for quasi-static and dynamic tests, respectively), Deformation at Skin Puncture (mm, related to tissue turgidity), Stress Relaxation (dimensionless, related to the visco-elastic behaviour), Magness-Taylor Resistance (N, related to fruit firmness), Fruit Mass (g, related to impact energy under free-fall conditions), Curvature Radius (mm, related to the stress accumulation in tissues), Fruit Deformability Modulus (N/mm, force to a specific fruit deformation), Rheological Behaviour (per mm, related to changes in the deformability modulus for an increasing load level), etc.

An important issue when using instrumental measurements for modelling purposes is to address their accuracy (proximity to a true value) and precision (repeatability). The accuracy for an instrument or instrumental procedure is difficult to assess when there is no certified reference available. Precision may be accounted through the standard deviation (S.D.) of replicates on the same specimen. In the case 
of individual fruits, intra fruit variability of mechanical properties has to be isolated from the precision of measurements, accounted as S.D. for local measurements. As a general measure, intra fruit variability lies between 10 and $20 \%$ of the average, i.e. 4 $\mathrm{N}$ for a $20 \mathrm{~N}$ firmness peach assessed as Magness-Taylor Resistance. The S.D. at a local level, referring to precision, lies between 6 and $10 \%$ of the average, i.e. $2 \mathrm{~N}$ for $20 \mathrm{~N}$ firmness peach assessed as Magness-Taylor Resistance. Under controlled conditions the variability in load level for laboratory tests is very low, with variations below $1 \%$ of the average.

The final input for modelling bruise susceptibility is usually a data matrix with as many rows as fruits tested. Columns in the matrix refer to a pool of mostly continuous variables, plus a Boolean variable indicating whether the fruit is actually bruised by a controlled test for several possible load levels ( 1 , bruised; 0 , nonbruised). Following European Union (EU) standards, a fruit is considered bruised for bruise sizes above $1 \mathrm{~cm}^{2}$ in pome fruit (apples and pears) and $0.5 \mathrm{~cm}^{2}$ in stone fruit. Each time a fruit is labelled as 1 (bruised), the bruise size exceeds the above numbers and, therefore, no further bruise categories need to be considered. On the other hand, a grading line should not exceed $10 \%$ of damaged fruit to be within the EU tolerance level. Within this context, the following logistic regression model is proposed.

\section{A logistic regression model for estimating bruise probability}

Let $y$ be the bruise response variable and $x_{1}, \ldots, x_{n}$ the remaining variables in the data matrix. Then the model is defined as:

$$
\ln \frac{p}{1-p}=f\left(x_{1}, \ldots, x_{n}\right)
$$

where, $p=P\left(y=1 \mid x_{1}, x_{2}, \ldots, x_{n}\right)$. Function $f$ may be a linear function $\beta_{0}+\beta_{1} x_{1}+$ $\ldots \beta_{n}, x_{n}$ of observations $\left(x_{1}, \ldots, x_{n}\right)$ and/or have non-linear terms.

The impact or load level applied to the fruit is always a variable $x_{i}$ present in the data matrix in our case because it is needed at each transfer point for the simulation.

\subsection{Data analysis}

Data analysis is performed mainly to remove the multicollinearity between $x_{i}$ 's. It is well-known that a model with several predictors has the potential for strong correlations among them, implying that some of them are redundant. Although treated extensively in the linear regression literature, multicollinearity has received very little attention in the logistic regression literature (Ryan, 1997). Since our predictors are all continuous, draftsman plots (an array of scatter plots) and the correlation matrix help us to detect this feature and we include it as part of the software program. 


\subsection{Model construction}

We first estimate the parameters of the logistic regression model by the method of maximum likelihood, based on a user's database. This is a fundamental requirement, since each variety, production area and procedure has a specific bruise susceptibility layout. Maximum likelihood estimators $\beta_{i}$ are obtained by maximising the logarithm of likelihood function $\mathscr{L}$ with respect to $\beta$,

$$
\begin{aligned}
\ln \mathscr{L} & =\sum_{i}\left\{y_{i} \ln \left(\frac{\mathrm{e}^{x_{j}^{\prime} \beta}}{1+\mathrm{e}^{x_{i}^{\prime} \beta}}\right)+\left(1-y_{i}\right) \ln \left(\frac{1}{1+\mathrm{e}^{x_{i}^{\prime} \beta}}\right)\right\} \\
& =\sum_{i} y_{i} x_{i}^{\prime} \beta-\sum_{i} \ln \left(1+\mathrm{e}^{x_{i}^{\prime} \beta}\right)
\end{aligned}
$$

where, $\beta^{\prime}=\left(\beta_{0}, \beta_{1}, \ldots, \beta_{n}\right)$ is the vector of coefficients and $x_{i}^{\prime}=\left(1, x_{i 1}, \ldots, x_{i n}\right)$ is the $i$-th observation on the corresponding $n$ explanatory variables. This vector would include components $x_{i j} \times x_{i k}$ when considering non-linear $x_{j} x_{k}$ interactions.

Unfortunately there is no analytical solution for $\beta_{i}$ but we may resort to using a Newton-Raphson iterative procedure. Each cycle in this procedure provides an updating formula given by:

$$
\beta^{(k+1)}=\beta^{(k)}+\left(\mathbf{X}^{\prime} \mathbf{W} \mathbf{X}\right)^{-1} \mathbf{X}^{\prime}(\mathbf{Y}-\mathbf{Y})
$$

where $\mathbf{Y}$ denotes the vector of response values, $\mathbf{X}$ denotes a matrix with each row given by $\left(1, x_{i}\right), \mathbf{Y}$ denotes the vector of estimated values at that iteration $p_{i}=$ $\mathrm{e}^{x_{i} \beta^{(k)}} /\left(1+\mathrm{e}^{x_{i} \beta^{(k)}}\right)$, and $\mathbf{W}$ denotes a diagonal matrix with elements $p_{i}\left(1-p_{i}\right)$. This formula is used until the estimates converge. The choice of the preliminary estimator is irrelevant. No single convergence criterion appears superior to the others. The SIMLIN $^{\circledR}$ software (see Pacios, 1999; Heradio, 2000 for its implementation) takes into account the change between successive steps in parameter estimates.

The maximum likelihood approach will generally perform well for large sample sizes. However, the estimators do not exist when the data are quasi-completely separated (Albert and Anderson, 1984). This means that there exist constants ( $\alpha_{0}$, $\left.\ldots, \alpha_{n}\right)$, with at least one $\alpha_{j} \neq 0, j=1, \ldots, n$, such that $\alpha_{0}+\alpha_{1} x_{1}+\ldots+\alpha_{n} x_{n} \geq 0$ for all $y_{i}=1$, and $\alpha_{0}+\alpha_{1} x_{1}+\ldots+\alpha_{n} x_{n} \leq 0$ for all $y_{i}=0$. In other words, there is a plane such that all $x$ 's corresponding to $y_{i}=1\left(y_{i}=0\right)$ are placed on one side (opposite side) of this plane. Although perfect prediction might be expected when data are separated, we are not going to encounter it very often because we have large sample sizes. Also, undefined expressions in the log likelihood are derived. To detect these troubles, SIMLIN ${ }^{\circledR}$ requires from the user the maximum number of iterations to perform. If this limit is exceeded without achieving the desired change, the process is terminated.

Model selection strategy is based on a backward stepwise elimination procedure. Backward selection should give an adequate model whenever the initial model is adequate (Christensen, 1997, p. 215). We start with the model that contains all the linear and quadratic effects ( $x_{i}$ and $x_{i} x_{j}$ terms, respectively). Quadratic terms allow the assessment of interaction between two independent variables. This preliminary 
model was judged to be adequate because of its interpretability and its consistency with model assumptions (Christensen, 1997), as suggested by the experts from the LPF. It is hierarchically well formulated since it contains all lower-order components of any term in the model. During the backward process, if a product term is found significant, the Hierarchy Principle requires that all lower-order components to remain in all further models considered (Kleinbaum, 1994).

The backward procedure eliminates at each stage the term in the model that has the largest $p$-value when we test that its coefficient equals zero, i.e. it removes the least significant variable that does not meet the level for staying in the model. Once a variable is removed from the model, it remains excluded. The process is repeated until no other variable in the model meets the specified level for removal. Note that this process is computationally costly in spite of examining a limited number of models, as in every stepwise process. As discussed by Hosmer and Lemeshow (1989) no research results have been reported that would suggest a reasonable value of $p$ in logistic regression. In any case, the sIMLIN ${ }^{(\mathbb{B})}$ software allows the user to specify the value of $p$ when operating in expert mode. In user mode the value of $p$ is automatically fixed at 0.1 .

Thus, we want to compare two (nested) models during the backward process, i.e. the hypothesis that the simpler model $\mathbf{M}_{0}$ holds against the alternative that the more complex one $\mathrm{M}_{1}$ holds, has to be tested. These models can be compared by comparing their deviances. The deviance in logistic regression corresponds to the residual sum of squares (SSE) in linear regression. Given a model $M$ with expected values $p_{i}$ its deviance $D_{\mathrm{M}}$ is defined to be:

$$
D_{\mathrm{M}}=-2 \sum_{i}\left\{y_{i} \ln \left(\frac{p_{i}}{y_{i}}\right)+\left(1-y_{i}\right) \ln \left(\frac{1-p_{i}}{1-y_{i}}\right)\right\} .
$$

Note that the first (second) term is considered zero when $\left.y_{i}=0 \quad y_{i}=1\right)$. Given that $\mathrm{M}_{1}$ holds, the statistic for testing that $\mathrm{M}_{0}$ holds is $D_{\mathrm{M}_{0}}-D_{\mathrm{M}_{1}}$, which is an approximate $\chi^{2}$ statistic, with df equal to the number of additional nonredundant parameters that are in $\mathbf{M}_{1}$ but not in $\mathbf{M}_{0}$ (Agresti, 1996). Since in our backward process we will always use a null hypothesis with only one parameter equal to zero, it implies $\mathrm{df}=1$. This test is equivalent to the $F$ test for comparing linear regression models.

Once we have a good model, sIMLIN $\left.{ }^{(}\right)$displays its output data: (a) the final maximum likelihood estimate $\beta$ of the parameters; (b) the estimated standard error of the parameter estimate, computed as the square root of the corresponding diagonal element of the estimated covariance matrix $\left(\mathbf{X}^{\prime} \mathbf{W X}\right)^{-1}$; (c) the $t$-value, computed as the parameter estimate divided by its standard error estimate, which is sometimes called the Wald statistic. Commonly encountered in any logistic regression package, this statistic is used in assessing the significance of a coefficient individually. Though it has the same form as a $t$-statistic, under the hypothesis that the coefficient is zero, it is asymptotically normally distributed with a large sample size required. Some authors have questioned its use (see e.g. Hosmer and Lemeshow, 1989, p. 17). 
We also compute predicted responses $p_{i}$ for each observation $i$ (estimated probability that fruit $i$ bruises), together with $95 \%$ (approximate) usual confidence intervals for probabilities $p_{i}$.

\subsection{Evaluating model predictive ability}

Even though a residual analysis is not appropriate here (Christensen, 1997, p. 131, 247) and diagnostic procedures are not, and maybe never will be, completely formalised (Ryan, 1997, p. 307), we deal with the problem as follows.

Since we are interested in predicting whether a fruit will or will not be bruised from the estimated bruise probability. Thus, a meaningful measure of the worth of the model would be the percentage of fruit in the data set that are classified correctly. Accordingly, we use the correct classification rate as a measure of the fit of the model. This statistic is potentially more informative than the $p$-value of a goodnessof-fit statistic (Ryan, 1997, p. 269). The classification of observations into groups is based on a cutoff value for $p_{i}$. All observations whose $p_{i}$ is greater than or equal to this value are classified as damaged fruit; otherwise, as undamaged fruit. Therefore, we compare observed values and discrete predicted values of the dependent variable. Should a comparison of observed values and continuous predicted values (probabilities) be wanted, a number of alternative measures or analogs of $R^{2}$ (coefficient of determination) have been proposed. Menard (2000) studied five of them, pointing out that there is as yet no consensus on how we should calculate and use those measures.

SIMLIN $^{(B)}$ computes a cutoff value that minimises the number of misclassifications, as recommended by many authors (e.g. Ryan, 1997). This seems quite reasonable since the costs of each type of misclassification are approximately equal. Sometimes the measure is called maximum correct classification rate (MCCR).

The classification table reports MCCR, sensitivity, specificity, false positive and false negative rates. The statistical significance of these rates can be assessed by using Huberty's procedure (Sharma, 1996). For example, suppose we are to test how good is the MCCR, i.e. whether MCCR is significantly greater than that due to chance. Let $m$ be the total number of observations, $O$ be the total number of correct classifications and $E=\left(m_{1}^{2}+m_{2}^{2}\right) / m$ be the expected number of correct classifications due to chance, where $m_{i}$ is the number of observations in group $i(i=1,2)$. Then, the statistic

$$
Z^{*}=\frac{(O-E) \sqrt{m}}{\sqrt{E(m-E)}},
$$

follows an approximately standard normal distribution.

We also need external validity, i.e. examination of the accuracy with which the logistic function can classify observations from another sample. For such purpose, one may use the Jackknife method (see e.g. Shao and Tu, 1995), that leaves out one observation at a time, estimates the model with the remaining observations, and classifies the left-out observation. Since it is necessary to run as many logistic 
regressions as there are observations, which is computationally quite cumbersome, SIMLIN ${ }^{(\beta)}$ uses an approximate and efficient procedure for obtaining one-step pseudoJackknife estimates of $\beta_{i}$ (see SAS, 1990). Then, $p_{i}$ and classification rates are computed accordingly.

Finally, the effect of an individual observation on the estimated parameter of the fitted model can be assessed, detecting whether it causes instability in that estimate. For each observation $i$ and each parameter $\beta_{j}$, the Dfbeta diagnostic is the standardised difference between $\beta_{j}$, computed with observation $i$, and $\beta_{j}^{(i)}$, computed without observation $i$. Instead of re-estimating the parameter every time an observation is deleted, the implemented procedure uses a one-step estimate to approximate $\beta_{j}^{(i)}$ from $\beta_{j}$ (see SAS, 1990). If such an influential observation is detected, the expert user may delete it and the procedure will be reinitiated.

\section{Simulation of a grading line}

The logistic regression model provides an estimation of fruit bruising probability $(p)$ as a function of variables $x_{i}$, one of which refers to the impact intensity recorded at a generic point of the line. Let $g(\cdot)$ be this function, i.e. $p=g\left(x_{1}, x_{2}, \ldots, x_{n}\right)$, where $x_{1}$ is assumed to be the impact intensity.

We then construct the simulation model of fruit moving along the grading line. The flowchart (Fig. 1) shows the algorithm to be repeated until the number of required outputs are yielded.

We start by generating values to characterise the fruit (variables $x_{2}, \ldots, x_{n}$, see Section 3) according to their distributions. For example, when these distributions are normal, the efficient polar generation method is used (Law and Kelton, 1991). These values characterise the fruit and once they are introduced into $g(\cdot)$, they provide bruise probability as a function of $x_{1}$. Fruit characterisation is kept constant along the grading line, i.e. we consider that the intrinsic characteristics of a fruit are not affected as it moves along the line.

At this stage, we virtually place the fruit at the starting point of the line. For the first transfer point, we simulate an impact $x_{1}$ from the discrete distributions previously yielded by the electronic fruits (see Section 2) using the inverse-transform method (Law and Kelton, 1991). Since we actually simulate an impact category from the database, its middle point is then entered into the logistic model for damage prediction. This process leads to a bruise probability $p^{\prime}$ when substituting the generated $x_{1}$ in function $g(\cdot)$. Finally, we determine whether or not the fruit is damaged at the transfer point in question by generating a value from a Bernoulli distribution of parameter $p^{\prime}$. If the fruit is not damaged, it passes to the next transfer point of the line and the previous steps for impact and damage generation are repeated until the fruit reaches the end of the line. Whenever any transfer point damages the fruit, the item is removed from the simulation, since the goal is to compute the percentage of the total amount of handled fruit that is bruised and not the number of bruises per fruit. The removal of a fruit from simulation does not affect damage occurrence for other items. Fruit to fruit impacts are taken into 


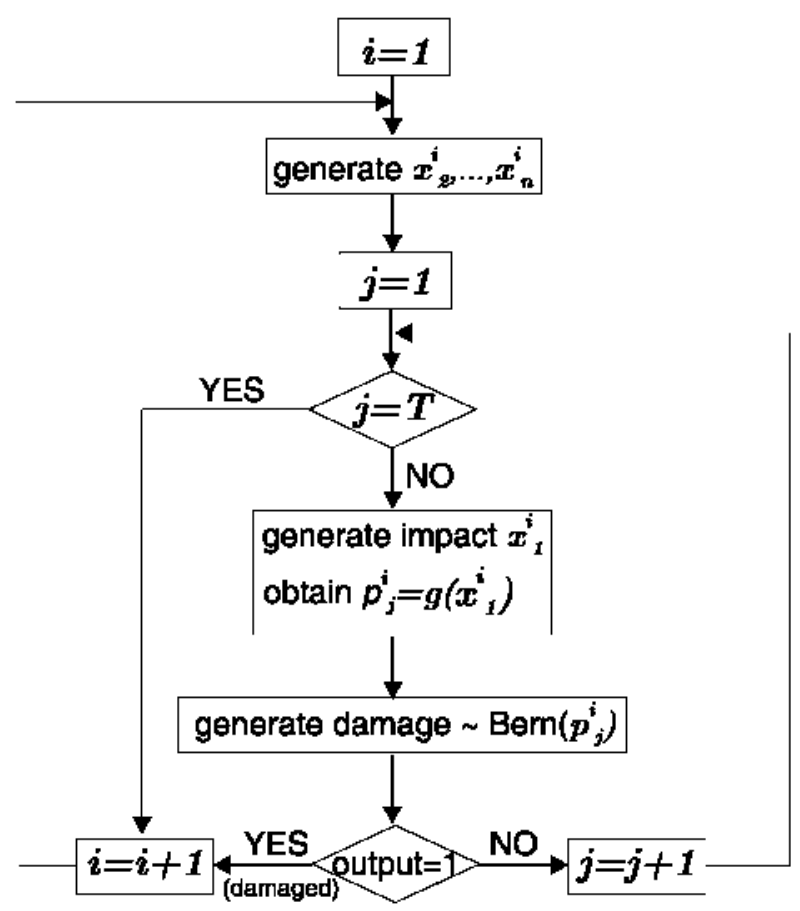

Fig. 1. Simulation of a grading line. Index $i$ records each piece of fruit $(i=1, \ldots, N)$ and index $j$ records each transfer point (from 1 to the total number $T$ of transfer points in the grading line).

account in the impact intensities recorded by the electronic fruits, obtained under real conditions. When a fruit is removed from simulation, the entire process is repeated for a new fruit entering the grading line, after increasing the total number of damaged fruit by one unit. By this means, the transfer point's contribution to damage at that specific location is assessed. This additional information is only acquired with the simulation of the whole grading line, and not with cach transfer point separately.

The final bruise probability for that line is approximated by $d / N$, where $d$ is the number of damaged fruit in $N$ repetitions of this process. Furthermore, the simulation algorithm can compute how much information must be collected in order to get a proper representation of the real system. Thus, the number $N$ of fruit to be simulated is not entered as an input; it is calculated by the SIMLIN $\left.{ }^{(}\right)$program itself after having set the required precision for the simulation output. This precision is given by a confidence interval, defined by its width and confidence level. See Ross (1990) (p. 97) for further details. 


\section{A real example: Sudanell peaches}

The following example is based on a variety of peaches called Sudanell. The input file has a row for each of 360 fruit, each row recording the following characteristics: Impact Intensity $\left(g \times \mathrm{m} / \mathrm{s}^{2}\right)$, Fruit Deformability Modulus $(\mathrm{N} / \mathrm{mm})$, Rheological Behaviour $\left(\mathrm{mm}^{-1}\right)$, Skin Resistance (N), Deformation at Skin Puncture (mm), Stress Relaxation (dimensionless), and Magness-Taylor Resistance of the Flesh (N). As mentioned in Section 2, the precision of measurement lies between 6 and $10 \%$ of the average. The limit for model prediction depends on the quality of those data coming from independent variables and references. Up to now, this limitation is far more important than the computing precision. This data set was derived from experiments carried out at LPF (Technical University of Madrid) and the Applied Edaphology and Biology Research Centre (Murcia, Spain), under the National Project PTR 94-0082: technology transfer of harvest and post-harvest techniques in Cooperatives of the Murcia region. A factorial design was applied to several precooling treatments as well as various post-harvest ripeness states of the fruit to generate as wide range of inherent susceptibility conditions to bruising as possible (García and Ruiz-Altisent, 1997). There were no missing data.

SIMLIN $^{\circledR}$ calculated that, for a $p$-value of 0.01 in the backward model selection procedure, this variety is described by a logistic regression model with three variables. Table 1 shows the main information. The deviance was 223 for this final model, compared with a deviance of 469 for a model with only one parameter (intercept).

Note the power of this model that has only three terms out of seven potential additive terms and $\left(\begin{array}{l}7 \\ 2\end{array}\right)=21$ interaction terms in the initial model (whenever there is no multicollinearity, which is checked initially by the program). This simplicity represented by parsimony of parameters is a desirable feature of any model. With a $p$-value equal to 0.05 , skin resistance $(\mathrm{N})$ and deformation at skin puncture $(\mathrm{mm})$ also enter the model. With $p=0.1$, there are obviously more variables in the model, even interaction terms. The experts from LPF agreed with the first model due to its simple interpretability, so its predictive ability is evaluated here.

Logistic regression output also includes the estimated probability for each fruit to be bruised, together with $95 \%$ confidence intervals for probabilities $p_{i}$. For reasons

Table 1

Logistic regression output (part I): maximum likelihood estimates $\beta$, of the parameters, estimated standard error of the parameter estimate and $t$-value

\begin{tabular}{lrlr}
\hline Term $i$ & \multicolumn{1}{c}{$\beta_{i}$} & Standard error & $t$-value \\
\hline Intercept & -13.56 & 2.07 & -6.54 \\
Impact intensity & 0.04 & 0.007 & 6.04 \\
Stress relaxation & 0.67 & 0.09 & 7.65 \\
Magness-Taylor resistance & -0.15 & 0.04 & -3.99 \\
\hline
\end{tabular}


Table 2

Logistic regression output (part II): values for Impact intensity, Stress relaxation and Magness-Taylor resistance for each fruit. estimated probability for each fruit and $95 \%$ confidence intervals (CI) for probabilities $p_{i}$

\begin{tabular}{cccccccc}
\hline Observation & $y$ & Impact Intensity & Stress Relaxation & Magness-Taylor Resistance & $p$ & $95 \%$ CI for $p$ \\
\hline 1 & 0 & 160.4 & 8.3 & 13 & 0.03 & $(0.01,0.08)$ \\
$\vdots$ & $\vdots$ & $\vdots$ & $\vdots$ & $\vdots$ & $\vdots$ & $\vdots$ \\
67 & 1 & 75.8 & 21.2 & 9 & 0.92 & $(0.83 .0 .96)$ \\
$\vdots$ & $\vdots$ & $\vdots$ & $\vdots$ & $\vdots$ & $\vdots$ & $\vdots$ \\
\hline
\end{tabular}

of space, take, as an example, observations 1 and 67 and see the corresponding rows in Table 2. Values for the three variables in the model are also provided.

Graphics of Dfbeta diagnostics for the three parameter estimates revealed that there was not any influential observation (all Dfbetas were less than 0.4 in absolute value).

The classification table (see Table 3) was built with a cutoff value of 0.35 . Also, MCCR $=84.4 \%$; sensitivity $=84.3 \%$ and specificity $=84.5 \%$, which are good results. Huberty's procedure gave $Z^{*}=11.52$, which is statistically significant at an alpha value of 0.05 . That is, the MCCR of $84.4 \%$ is significantly higher than that expected by chance alone $(54.2 \%)$.

Having evaluated the model for this species, we must fix the specific state of the fruit entering the line. A Sudanell peach is different at the beginning of the season from at the end, but it also depends on the harvest and post-harvest treatments. Thus, this characterisation is carried out by telling the program which parameters define the probability distribution of $x_{2}, \ldots, x_{n}$ we are going to generate from. Note that the technique used by Bollen and Cox (1991) and Bollen (1993) is far less flexible, since it has to adjust the coefficients of the logistic regression model separately for each fruit characterisation and each impact surface.

In this example, the hypothesis of a normal distribution for Stress Relaxation cannot be rejected by usual goodness-of-fit tests. We decided to set the sample values $\mu_{\mathrm{ST}}=14.7$ and $\sigma_{\mathrm{ST}}=4$ (dimensionless), since they are the maximum likelihood estimators for this distribution. Magness-Taylor Resistance behaved in a similar manner, with sample values $\mu_{\mathrm{MT}}=11.3$ and $\sigma_{\mathrm{MT}}=6.1(\mathrm{~N})$. In the next section we will perform some experiments changing the state of the fruit demonstrating more

Table 3

Classification table: number of fruit correctly and incorrectly classified into groups of damaged and undamaged fruit

\begin{tabular}{llc}
\hline Observed & Predicted & \\
\cline { 2 - 3 } & Damaged & Not damaged \\
\hline Damaged & 108 & 20 \\
Not danaged & 36 & 196 \\
\hline
\end{tabular}


extreme cases. Note that $\mu$ and $\sigma$ refer to the average and standard deviation of the population to be simulated, respectively.

Next, we have to specify the grading line we are going to work with. Here we will work with a real design comprising the following elements in the indicated order: twist chain dumper (E1), conveyor belt (E2), sorting table (E3), vacuum generator, to remove the peach fuzz (E4), conveyor belt (E5), electronic sizer (E6), conveyor belt (E7), and packaging area (E8). These eight elements have seven transfer points. The line has to be validated by the program according to constraints supplied by experts, e.g. the sizer must precede the packaging area. This ensures that the line introduced by the user is well-defined. Otherwise, the posterior steps would be invalid. SIMLIN ${ }^{(B)}$ includes a database generated on real commercial lines by means of electronic fruits, IS-100 type, within the scope of the above mentioned National Project (García et al., 1996). From this database, SIMLIN ${ }^{(B)}$ extracts the seven probability distributions of impact intensity at each transfer point (see Table 4). The transfer point at row $i(i=1, \ldots, 7)$ is that from $\mathrm{E} i$ to $\mathrm{E}(i+1)$.

We set a precision for the simulation output defined by a confidence interval with a high confidence level $(99 \%)$ and a short width $(0.01)$. From this precision, SIMLIN ${ }^{(B)}$ computes the number $N$ of fruit to be simulated. In this example, $N=51833$.

A wide variety of outputs can be obtained using SIMLIN ${ }^{(\mathbb{E}}$. One of them shows the total number of damaged fruit, 34940 fruits $(67.4 \%)$. Though extremely high, this fact has been repeatedly observed in the five cooperatives tested within the mentioned National Project.

The program plots the number of fruit processed at each transfer point and how many were damaged. The number of fruit processed is decreasing along the line because the fruit is removed from the simulation whenever any transfer point damages it, see Section 4. For that reason, it is important to plot the percentage of damaged fruit at each transfer point with respect to the total number of damaged fruit in the line, and also, with respect to the total number of processed fruit at that transfer point. The information given by the latter plot is summarised in Table 5 .

The first transfer point (E1-E2) is the most critical point along the line. This connection between the dumper and a conveyor belt would have damaged $58 \%$ of the fruit processed there. The sixth transfer point between the electronic sizer (E6) and a conveyor belt (E7) would have damaged 14.6\%. By comparison, the remaining

Table 4

Impact distributions at each transfer point

\begin{tabular}{lllll}
\hline Transfer point & Impact $\leq 50 \mathrm{~g}$ & $50-100 \mathrm{~g}$ & $100-150 \mathrm{~g}$ & $150-200 \mathrm{~g}$ \\
\hline 1 & 0.27 & 0.21 & 0.35 & 0.17 \\
2 & 0.86 & 0.14 & 0 & 0 \\
3 & 0.37 & 0.63 & 0 & 0 \\
4 & 0.85 & 0.15 & 0 & 0 \\
5 & 0.87 & 0.13 & 0 & 0 \\
6 & 0.2 & 0.47 & 0.16 & 0.17 \\
7 & 0.82 & 0.14 & 0.04 & 0 \\
\hline
\end{tabular}


Table 5

Percentage of damaged fruit at each transfer point, with respect to the total number of fruit processed at that point

\begin{tabular}{ll}
\hline Transfer point & Damaged fruit $(\%)$ \\
\hline 1 & 58 \\
2 & 1.7 \\
3 & 3.7 \\
4 & 1.2 \\
5 & 1.1 \\
6 & 14.6 \\
7 & 1.5 \\
\hline
\end{tabular}

transfer points would hardly damage any fruit. This demonstrates the need to substitute the twist chain dumper for another design, and to replace the conveyor belt (E7) by a less aggressive one, such as coated rollers. Section 6 deals with these possibilities.

In addition, the number of damaged fruit for different levels of each $x_{i}$ in the model are recorded. It is then possible to determine which type of intrinsic characteristics of the fruit enhances bruising. Fig. 2 shows the influence of Stress Relaxation on damage occurrence along the line. For example, it can be seen that most simulated fruit had values less than 19 (see the left-hand bars at each interval). The interval $(13.8,17)$, represented in Fig. 2 by its middle point 15.4 , indicates that $29.9 \%$ of the simulated fruits were generated within that interval and $24 \%$ of them were damaged (see the third white bar from the left). At the next three intervals of higher Stress Relaxation, almost all the fruits were damaged. Therefore, a fruit within these values of Stress Relaxation is the most sensitive to damage.

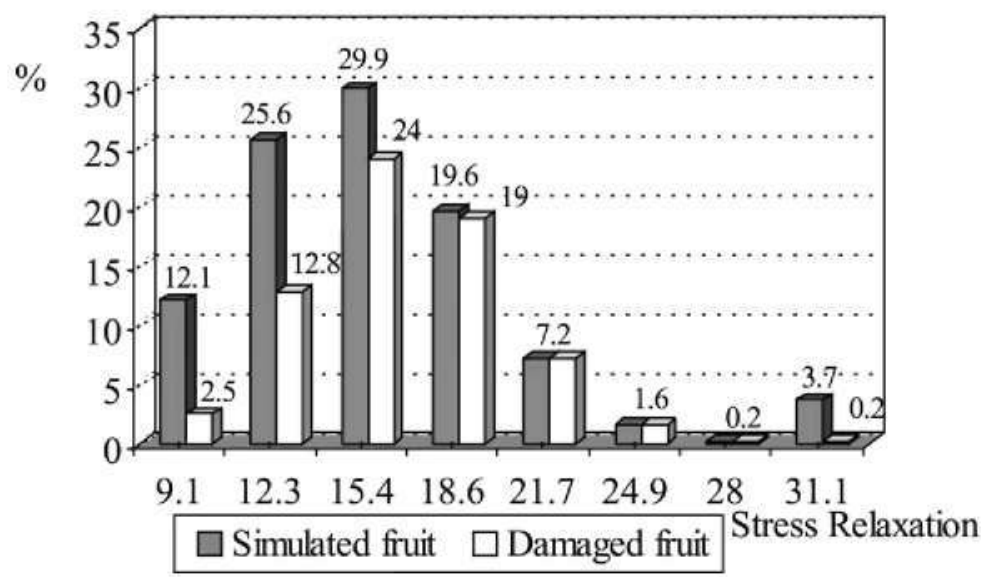

Fig. 2. Percentage of simulated (shown in black) and damaged (in white) fruit for different values of variable Stress Relaxation (in $X$ axis). 
There is an analogous plot for the other characteristic, Magness-Taylor Resistance.

The next section is devoted to the improvement of the line to achieve bruise levels within the EU limits (at most, $10 \%$ of damaged fruit).

\section{Searching for optimal operating conditions in fruit grading lines}

The high percentage of damaged fruit $(67.4 \%)$ obtained with the grading line described in Section 5, shows that there can be a need for significant improvement.

Dumping is one of the most dangerous transfer points in almost every line. In our example, it has damaged $58 \%$ of the fruit. Thus, we start by changing the twist chain dumper to a less aggressive one, a box dumper. This new grading line will be referred as line-2, while the initial line will be called line-1.

Using the same precision as in the previous section (a width of 0.01 for the $99 \%$ confidence interval), this line yields 29231 damaged fruits over a total of $N=56785$ fruits, i.e. $51.5 \%$.

The number of damaged fruit at the new first transfer point (now, referred as to $1^{\prime}$ ) has decreased from 58 to $48.9 \%$, but it remains a critical point in the line together with the sixth transfer point, the electronic sizer-conveyor belt. Thus, we design from line-2 a third line (line-3) substituting at the sixth transfer point the conveyor belt for coated rollers, to decrease the number of damaged fruit. This new transfer point is referred as to $6^{\prime}$. Table 6 shows the probability distributions of impact intensity at transfer points $1^{\prime}$ and $6^{\prime}$, which substitute 1 and 6 , respectively in Table 4 . The final configuration achieved with line-3 corresponds to some of the latest technical designs and, therefore, it could be seen as near the actual limit for lowest machinery aggressiveness. Nonetheless, in this line the percentage of damaged fruit is $42.3 \%$ ( 26704 over $N=63174$ fruits). Note that this value greatly exceeds the EU tolerance level ( $10 \%$ of damaged fruit). The first transfer point still records $25.7 \%$ of damaged fruit while it is $2.8 \%$ at the sixth transfer point. We then conclude that due to the properties of the fruit used in the simulation it is impossible to attain the EU tolerance level.

The solution is to select new states of fruit for simulation, using the logistic model as a sensitivity tool. Predicted bruise probability of the fruit to be handled should not exceed 0.1 for $25 \mathrm{~g}$ (minimum impact load to be generated during simulation, see Section 4) when the EU tolerance level is to be attained for a grading line. This is a

Table 6

Impact distributions at new transfer points

\begin{tabular}{lllll}
\hline Transfer point & Impact $\leq 50 \mathrm{~g}$ & $50-100 \mathrm{~g}$ & $100-150 \mathrm{~g}$ & $150-200 \mathrm{~g}$ \\
\hline $1^{\prime}$ & 0.77 & 0.12 & 0.11 & 0 \\
$6^{\prime}$ & 0.79 & 0.21 & 0 & 0 \\
\hline
\end{tabular}

Transfer points $1^{\prime}$ and $6^{\prime}$. which substitute 1 and 6 respectively in Table 4. 
necessary condition although it is not sufficient. To find optimal properties of the fruit for simulation, values for Magness-Taylor Resistance and Stress Relaxation may be varied within the logistic model, testing the effect on final bruise probability at each impact intensity $(25 g, 75 g, 125 g, 175 g)$.

Thus, bruise probability may be decreased by increasing the mean $\left(\mu_{\mathrm{MT}}\right)$ of Magness-Taylor Resistance distribution, since its parameter estimate in the logistic model is negative. However, we should decrease the mean ( $\left.\mu_{\mathrm{Rs}}\right)$ of the Stress Relaxation distribution to decrease bruise probability, since the coefficient in the logistic regression for this variable is positive. After several trials, $\mu_{\mathrm{MT}}=20 \mathrm{~N}$ and $\mu_{\mathrm{RS}}=14$ (dimensionless) were selected for new simulations. These values correspond to a harder consistency of fruit than that of the sample values, achieved by harvesting earlier in the season, yet within a high organoleptic range (Alavoine et al., 1981). Selecting even harder consistencies of fruit (for example $\mu_{\mathrm{MT}}=40 \mathrm{~N}$ ) is not advisable because it could be far from consumer acceptance.

In addition to specifying the average conditions of the fruit state parameters, it is necessary to define the degree of dispersion for both Magness-Taylor Resistance and Stress Relaxation. To illustrate this, we use two characterisations of the fruit: (1) homogeneous fruit (with little dispersion), i.e. $\sigma_{\mathrm{MT}}=3 \mathrm{~N}$ and $\sigma_{\mathrm{Rs}}=1$ (dimensionless); and (2) heterogeneous fruit (more dispersion), with $\sigma_{\mathrm{MT}}=6 \mathrm{~N}$ and $\sigma_{\mathrm{RS}}=4$ (dimensionless). Recall that we will use the mean values mentioned above: $\mu_{\mathrm{MT}}=20$ $\mathrm{N}$ and $\mu_{\mathrm{RS}}=14$ (dimensionless). Therefore, it amounts to having six new experiments, since we have two types of fruit and three different grading lines.

Fig. 3 shows the simulation results for both groups in the three lines. It shows the percentage of damaged fruit from simulation and the associated $99 \%$ confidence interval (width of 0.01 ) in $X$-axis, as well as the number $N$ of fruit simulated at each case to obtain the required precision.

As far as the two kinds of fruit are concerned; as expected the homogeneous fruit yields better results in all grading line designs than the heterogeneous group.

As regards the lines, there is little difference between homogeneous and heterogeneous fruit within line-1, due to the high percentage of damaged fruit at the first point (transfer between dumper and a conveyor belt). The difference in damaged fruit is less than $3 \%$. Substituting the dumper and thereby obtaining line-2, this difference increases. Furthermore, there is a significant reduction in the percentage of damaged fruit for both groups, but it is not sufficient to reach the EU tolerance level.

When handling fruit in line-3, which has coated rollers instead of the conveyor belt of line-2, the difference between homogeneous and heterogeneous fruit is even more marked. A comparison of line- 2 and line- 3 shows that the results for line- 2 with the homogeneous group are approximately the same as for line- 3 using the heterogeneous one. More interestingly, in line- 3 homogeneous fruit reaches damages below $10 \%$, obtaining an important reduction of the percentage of damaged fruit at each transfer point. For example, the first transfer point damaged $4.1 \%$ of the fruit processed there. The remaining transfer points had percentages of damaged fruit between 0.5 and $1.5 \%$. 


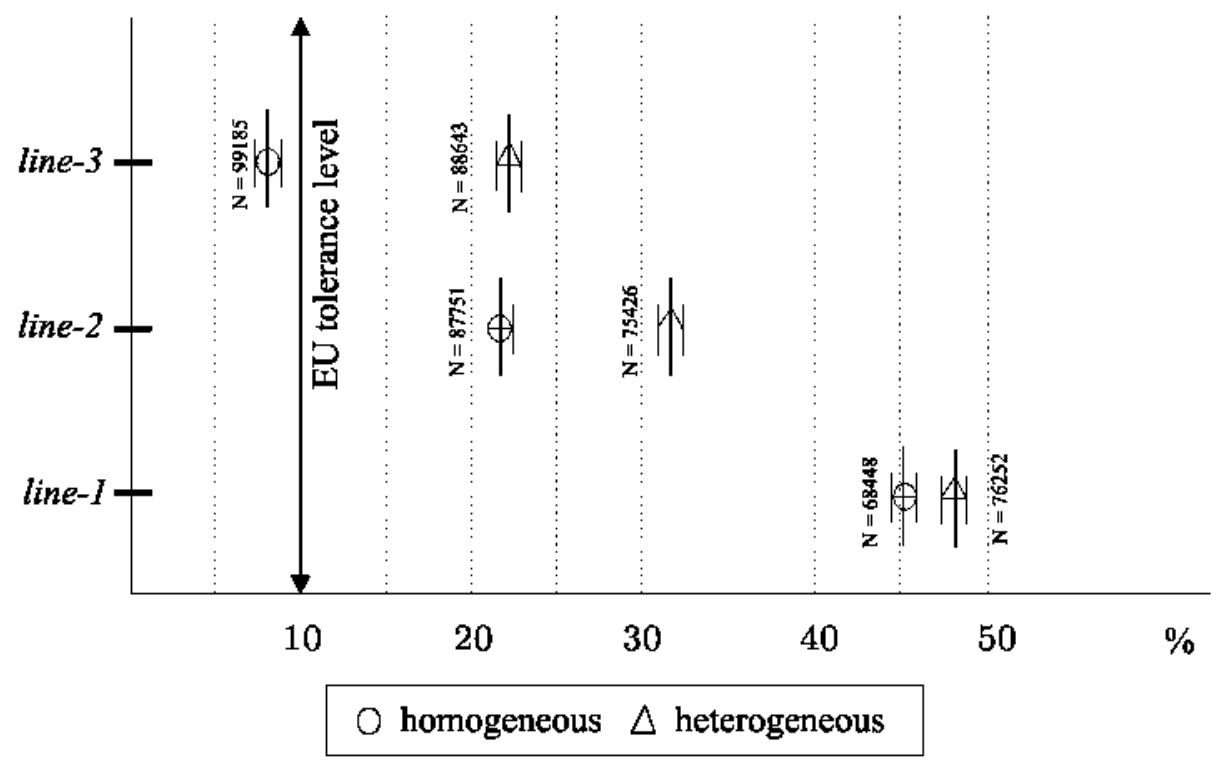

Fig. 3. Percentage of bruised peaches from simulation and the associated $95 \%$ confidence interval. The number $N$ of fruit simulated at each case to obtain the required precision is shown.

Then, it is only possible to reach the EU tolerance level for damages when (1) handling homogeneous fruit (within consumer acceptance range), and (2) on line-3.

\section{Conclusions}

The current prediction and simulation models can be used to describe the flow of fruits along the grading line, cvaluating whether or not there is a significant impact at each transfer point. Furthermore, it is possible to estimate the percentage of damaged fruit at the end of the line as a function of its intrinsic susceptibility. The accumulation of data for a large number of fruit makes it possible to draw conclusions on line operation for any design as a function of the characteristics of the handled fruit, provided the establishment of a desired confidence level has been stated for the simulated data.

The final conclusion of our experiments is that we are able to address the design improvements in the line as well as the optimal operating conditions of fruit for the improved line. The use of SIMLIN ${ }^{\circledR i}$ to cut quality losses in fruit due to mechanical damage is helpful to mect EU standards for damages.

Morcover, $\operatorname{sim} L i N^{\left({ }^{(1)}\right.}$ is a flexible tool. Any new data set can be readily incorporated. New line elements for the grading line can be defined. Impact probabilities for various intensity ranges can be modified. Impact Intensity distribution may be used as continuous, once its corresponding simulation method 
is implemented. Probability distributions that characterise the intrinsic susceptibility of the fruit can also be varied.

\section{Acknowledgements}

This work was supported by Technical University of Madrid, Spain, Project No. 8109 , and coordinated by Margarita Ruiz-Altisent. The fourth author was supported by Junta de Extremadura, Spain, Project No. IPR00A075. We wish to thank Óscar Pacios and Rubén Heradio for the software implementation, and Francisco García García for his help in providing the real data used in this paper.

\section{References}

Agresti, A., 1996. An Introduction to Categorical Data Analysis. Wiley,

Alavoine. F., Crochon. M.. Fady. C.. Fallot. J.. Moras. P., Peach, J.C.. 1981. La Qualité Gustative des Fruits. Méthodes Pratiques d'Analyse. CEMAGREF.

Albert. A. Anderson, J.A., 1984. On the existence of maximum likelihood estimates in logistic models. Biometrika 71, 1-10.

Barreiro, P., Steinmetz, V., Ruiz-Altisent, M.. 1997. Neural bruise prediction models for fruit handling and machinery evaluation. Computers and Electronics in Agriculture 18, 91-103.

Bollen. F., 1993. Determination of the probability of bruising. Australasian Postharvest Conference.

Bollen, A.F.. Cox, N.R., 1991. A technique for predicting the probability of bruising for use with an instrumented sphere. American Society of Agricultural Engineers, 1991 International Winter Meeting.

Chen. P.. Yazdani. R., 1991. Prediction of apple bruising due to impact on different surfaces. Transactions of the ASAE 34 (3), 956-965.

Christensen. R., 1997. Log-Linear Models and Logistic Regression. second ed. Springer, New York.

Garcia, F., Ruiz-Altisent, M.. 1997. Effects of precooling and degreening treatments on the susceptibility of peach and citrus to handling damage. In: Proceedings of 5th International Symposium on Fruit. Nut and Vegetable Production Engineering. California (USA).

Garcia, J.L., Riquielme, F.. Ruiz-Altisent. M., Barreiro, P.. 1996. Study of Grading lines for stone fruits and citrus using two instrumented spheres. In: Some Cooperatives in the Region of Murcia (Spain). Proceedings of AgEng'96 Conference on Agricultural Engineering, Paper 96F-038.

Garcia. F.. Barreiro, P., Ruiz-Altisent, M. Bielza. C., 2000. Bruise simulation in peach grading lines as a tool for designing improvement strategies. In: Proceedings of AgEng 2000 Conference on Agricultural Engineering. Warwick (UK), 36-37. Full paper (8 pages) available at http//irul6.iru.etsia.upm.es.

Garcia-Ramos, F.J., Barreiro, P., Ruiz-Altisent. M., Ortiz-Cañavate. J., Gil-Sierra, J., Homer, L., 2002. A procedure for testing padding materials in fruit packing lines. Transactions of the ASAE 45 (3), 751 757.

Heradio, R., 2000. Modelo de Regresión Logística para Caracterización de Especies de Frutas. Proyecto Fin de Carrera (Degree Project), School of Computer Science, Technical University of Madrid. http $/ /$ www.fi.upm.es/-servicios/biblioteca/index.html.

Hosmer, D.W, Lcmeshow, S.. 1989. Applied Logistic Regression. Wiley, New York.

Jones, C.S., Holt, J.E.. 1991. A model to predict danage to horticultural produce during transport. Journal of Agricultural Engineering Research 50, 259-272.

Kampp, J.. Pedersen, J., 1990. Quality of imported and domestic fruits and vegetables in the Danish retail trade with special reference to mechanical damage. In: Proceedings of 22nd International Conference on Agricultural Mechanization.

Kleinbaum, D.G.. 1994. Logistic Regression. Springer. New York.

Law. A.M., Kelton. W.D., 1991. Simulation Modeling and Analysis, second ed.. McGraw-Hill, Singapore. 
Menard, S., 2000. Coefficients of determination for multiple logistic regression analysis. The American Statistician 51 (1), 17-24.

Miller, W.M., Wagner, C.. 1991. Florida citrus packing line studies with an instrunented sphere. Applied Engineering in Agriculture 7 (5), 577-581.

Ortiz-Cañavate, J., Garcia-Ramos, F.J., Ruiz-Altisent. M.. 1999. Testing equipment for the improvement of mechanical devices to minimize damage to fruit in commercial packing lines. In: 27 International Synposium on Agricultural Engineering. Actual Tasks on Agricultural Engineering. ISBN 953-6135$26-4,223-229$.

Pacios, O., 1999. Simulador de Lineas de Clasificación. Proyecto Fin de Carrera (Degree Project), School of Computer Science, Technical University of Madrid. http//www.fi.upm.es/servicios/biblioteca/ index.html.

Prussia, S.E., Hung, Y.C., Shewfelt. P.L.. Jordan. J.L., 1987, Latent Damage in Apple and Peaches. ASAE paper no. 87-6520.

Ross, S.M.. 1990. A Course in Simulation. Macmillan Publishing Company, New York.

Ryan, T.P., 1997, Modern Regression Methods. Wiley, New York.

Sargent. S.A., Brecht, J.K.. Zollner. J.J.. 1992. Instrumented sphere impact analysis of tomato and bell pepper packing lines. Applied Engineering in Agriculture 8 (1), 76-83.

SAS Institute, Inc., 1990. SAS/STAT User`s Guide. Version 6. fourth ed.. vol. 2. Cary, NC. SAS Institute, Inc.

Shao. J., Tu. D.. 1995. The Jackknife and Bootstrap. Springer, New York.

Sharma, S., 1996. Applied Multivariate Techniques. Wiley, New York.

Timm, E.J., Brown, G.K., Brook, R.C., Schulte, N.L., Burton. C.L., 1991. Impact bruise estimates for onion packing lines. Applied Engineering in Agriculture 7 (5). 571-576. 